\title{
Prognostic significance of mean platelet volume in lung cancer patients: A meta-analysis
}

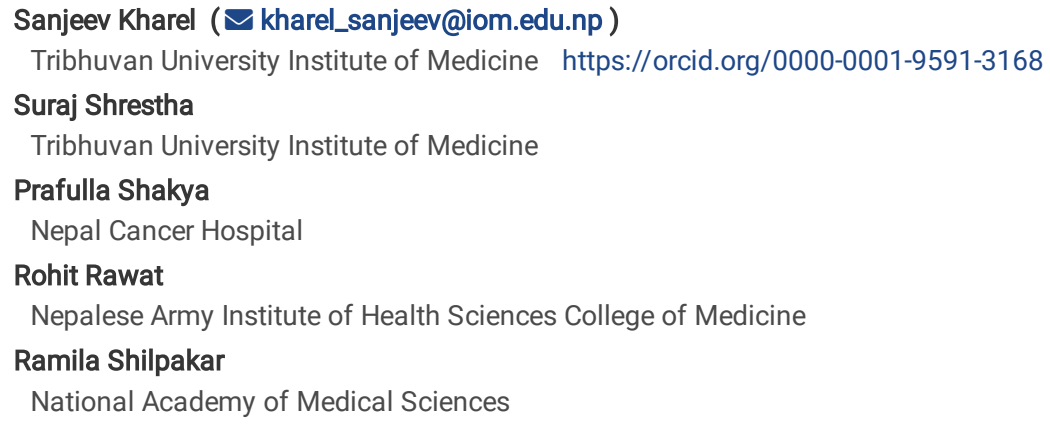

Keywords: Lung Cancer, Meta-analysis, Mean platelet volume, prognosis.

Posted Date: July 19th, 2021

DOI: https://doi.org/10.21203/rs.3.rs-678719/v1

License: (c) (i) This work is licensed under a Creative Commons Attribution 4.0 International License. Read Full License

Version of Record: A version of this preprint was published at Journal of International Medical Research on March 1st, 2022. See the published version at https://doi.org/10.1177/03000605221084874. 


\section{Abstract}

Background Lung cancer is the most prevalent cancer globally with a grim prognosis alongside a very high number of cancer-related deaths. Mean platelet volume (MPV) is the measure of platelet size and is considered a surrogate marker of platelet activation. Low MPV indicates exhausted platelets causing worse outcomes in cancer patients. As the correlation between platelet count/platelet size and lung cancer prognosis still remains a topic of debate, this metaanalysis was done to comprehensively evaluate the prognostic significance of MPV among lung cancer patients.

Methods A systematic search of electronic databases PubMed, Embase, and Google scholar and additional sources for relevant studies were done with no language restrictions from inception to 7th May 2021. Overall Survival (OS) and Disease-Free Survival (DFS)/Progression-Free Survival (PFS) and hazard ratio (HR) with 95\% Confidence Interval (Cl) were pooled to evaluate the relation of MPV with OS/DFS. Subgroup analysis based on cancer type, clinical stage, sample size, median age, cut-off value, and study region was done to identify the cause of significant heterogeneity.

Results Eleven studies with 2421 lung cancer patients were included in our analysis. Our analysis showed no significant association between MPV levels with OS (H.R.:1.07, 95\%C.I.:0.84-1.35, p = 0.60) and DFS/PFS (HR:1.04, 95\%C.1.:0.68-1.60, $p=0.84)$. Under subgroup analysis, studies conducted in countries other than China (HR:1.53, 95\% C.I. $=1.14-2.03, \mathrm{p}<0.001, \mathrm{I} 2=42.11 \%)$ and studies with advanced-stage lung cancer patients (HR: $1.84,95 \%$ C.I. $=1.19-2.82, \mathrm{p}-\mathrm{value}$ $=0.01,12=0 \%$ ) showed significant association between MPV levels and worse DFS/PFS.

Conclusion Pretreatment MPV levels did not show prognostic significance except in advanced lung cancer cases. Large multicentric studies with large samples and long follow-up times are necessary.

\section{Background}

Lung cancer is the most prevalent cancer globally with a very high number of cancer-related deaths in both men and women. ${ }^{1}$ The prognosis of lung cancer is grim with a five-year survival rate well below $15 \%$ despite advancements in radical surgery, radiotherapy, chemotherapy, and targeted therapy or immunotherapy. ${ }^{2}$

Various novel biomarkers that are able to predict the prognosis of lung cancer have been identified and include carcinoembryonic antigen, cytokeratin-19 fragments, squamous cell carcinoma antigen, progastrin-releasing peptide, tumor M2-pyruvate kinase, and C-reactive protein. However, these prognostic biomarkers are not included in the routine test in the majority of the patients with lung cancer owing to their high costs. ${ }^{3}$ Mean platelet volume is an inexpensive and potential prognostic marker that has been explored in a variety of cancers including lung cancer.

Mean platelet volume (MPV) is the measure of platelet size and is considered a surrogate marker of platelet activation. ${ }^{4}$ The large-sized platelets are more reactive and likely to aggregate more, thus are easily perishable. Cancer-associated increase in platelet activation and subsequent exhaustion is a plausible hypothesis that explains the decrease in platelet size in cancer patients including lung cancer. ${ }^{4,5}$ Thus, a low MPV indicates exhausted platelets with potentially tumor growth-promoting cytokines causing worse outcomes in cancer patients. ${ }^{6}$

Various studies have explored the potential role of MPV largely as a prognostic and predictive biomarker among patients with cancer such as gastric, bladder, renal, endometrial, non-small cell lung cancer (NSCLC), and hepatocellular carcinoma. ${ }^{7-12}$ However, the correlation between platelet count/platelet size and lung cancer prognosis still remains a topic of debate as some studies have identified that MPV is a poor prognostic factor in NSCLC, while some suggest that MPV has no association with lung cancer. ${ }^{11,13-15}$ Thus, there is a need for robust analysis to investigate the prognostic value of MPV in the survival rate among patients with lung cancer. We aimed to perform a meta-analysis to comprehensively evaluate the prognostic significance of MPV to one of the most notorious cancers with the highest mortality in the world.

\section{Methods}

\section{Data sources and Search Strategies}

Electronic Databases like PubMed, Embase, and Google Scholar were searched for the identification of relevant studies with no language restrictions from inception to 7th May 2021. The search used combinations of the terms "Mean Platelet Volume", "MPV" and "Lung Neoplasms", "Lung Cancer", "Carcinoma, Non-Small Cell Lung", "Carcinoma, Small Cell" as both medical subject headings and keywords with an appropriate Boolean operation. The detailed search strategy used is available in supplementary file 1. Furthermore, we checked the reference lists of all the included studies and also studies included in previous reviews to identify additional studies, if any. A Grey literature search was done using Google Scholar and Open Grey. Furthermore, preprint servers and thesis repositories were also searched. Full texts were requested from the corresponding author via mail and ResearchGate. This meta-analysis is reported according to the PRISMA (Preferred Reporting Items for Systematic Reviews and Meta-Analyses) guidelines.

\section{Selection Criteria}

The criteria for inclusion of studies in this meta-analysis are: Studies providing 1) data on the prognosis of patients diagnosed with lung cancer pathologically 2) pretreatment mean platelet volume measured 3) hazard ratios (HR) and $95 \%$ confidence intervals (Cl) directly or providing sufficient information from which these values can be estimated 4) relationship of survival outcomes including overall survival (OS) or/and disease-free survival (DFS)/Progression-Free Survival (PFS) with Mean Platelet Volume.

\section{Exclusion criteria}


The following studies were excluded: 1) Letters, reviews, experimental studies, case reports, conference abstracts, and duplicated studies. 2) Non-human studies. 3)Studies that did not provide enough data to calculate the HR with its $95 \% \mathrm{Cls}$.

\section{Data Extraction and Quality Assessments}

Two independent authors (SK and SS) reviewed original articles and selected the articles as per the set eligibility criteria. Any discrepancies during the selection process were resolved through discussion with a third reviewer (PS). A data extraction spreadsheet was created on Microsoft Excel version 2013 (MicrosoftCorp, Redmond, WA, USA) to extract the data under different headings; Author, Publication Year, Study Region, Study Design, Age (median age of sample), Sample size, Follow up durations in months (Median/Range), Treatment status of patient, Clinical stage of cancer, Cut-off value of MPV, Cancer type, Hazards ratio (HRs) with 95\% Cls for Overall Survival (OS) and Disease-Free Survival (DFS)/Progression-Free Survival (PFS) and New-Castle Ottawa Scale (NOS) scores. HRs from the multivariate analysis were preferred than from univariate analysis if both were given because of the advantage of multivariate analysis to exclude correlated confounding factors. HRs from multivariable analyses were extracted when available. Otherwise, HRs from univariable analyses were extracted or estimated from Kaplan-Meier survival curves as described by Parmar and colleagues. ${ }^{16}$ The value estimated from these curves was confirmed by requesting the value of HR from the corresponding author of the concerned study. DFS and PFS were considered the same in the analysis. The Newcastle-Ottawa Scale was used for the quality assessment of each study and described under three headings: selection (5), comparability (2), and exposure (3). ${ }^{17}$ Two authors independently assessed the study while any disagreements were solved through discussion among each other and the third author. Studies with scores of 6 or higher were considered qualified for inclusion and studies with scores more than 7 were considered as high-quality studies.

\section{Data synthesis and statistical analysis}

All analyses were performed by using STATA V.16.0 (STATA). HR with 95\% Cls was used to evaluate the relationship between MPV value and lung cancer. Statistical heterogeneity was assessed using the Cochrane Q-test and the $\mathrm{I}^{2}$ statistic, with values of $\mathrm{P}<0.1$ or $\mathrm{I}^{2}>50 \%$ being considered as indicative of significant heterogeneity. ${ }^{18}$ Random effect model (DerSimonian-Laird method was applied in case of significant heterogeneity. Otherwise, a fixed-effect model was used to pool HR. ${ }^{19}$ Subgroup analysis based on cancer type, clinical-stage, sample size, median age, cut-off value, and study region was done to identify the cause of significant heterogeneity.

Moreover, a sensitivity analysis was performed by omitting every single study sequentially to check the stability robustness of the pooled outcomes. Publication bias of literature was estimated by Begg's correlation test and Egger's linear regression tests. The p-value of $>0.05$ was indicative of no significant publication bias along with the observation of symmetry of the funnel plot. ${ }^{20,21}$

\section{Results}

\section{Study characteristics}

A flowchart demonstrating the details of the study selection according to the PRISMA guidelines is shown in Fig. 1. A total of 85 studies were obtained through database searches. First, we removed 26 duplicate articles and the remaining articles were screened by titles and abstract. The remaining 31 articles with full text after screening were assessed as per the eligibility criteria. Finally, 11 full-text articles containing 2421 lung cancer patients were included for the meta-analysis. ${ }^{11,15,22-30}$

The characteristics of the included studies are given in Table 1. All the included studies were published from 2014 to 2020 and were retrospective studies. Five studies are from China ${ }^{8,24-26,29}$, three from Turkey ${ }^{15,22,23}$, two from Japan ${ }^{11,30}$ and one study from Korea ${ }^{28}$ respectively. A study by Shi et al. 2018 had two datasets for HRs for patients with adenocarcinoma and squamous cell carcinoma. ${ }^{24}$ Similarly, survival outcomes were described among NSCLC patients in 9 studies, SCLC patients in one study while both NSCLC + SCLC in one study. In our analysis, five studies had patients with advanced stages of lung cancer while 7 studies had mixed (both early and advanced) stages of lung cancer patients. Most of the patients in the included studies were elderly with a median age of patients above 60 years. While five studies used a cut-off value of MPV $<10 \mathrm{fL}$ while the other 5 used a cut-off value of MPV $>10 \mathrm{fL}$ while in the remaining study, it was not mentioned. Lastly, the quality of study assessed by the NOS scale ranged from 6 to 8 as shown in Table 2 . 
Table 1

Characteristics of the included studies in the analysis

\begin{tabular}{|c|c|c|c|c|c|c|c|c|c|c|}
\hline Authors & $\begin{array}{l}\text { Publication } \\
\text { Year }\end{array}$ & $\begin{array}{l}\text { Study } \\
\text { Region }\end{array}$ & Study design & $\begin{array}{l}\text { Age } \\
\text { (median } \\
\text { age) }\end{array}$ & $\begin{array}{l}\text { Sample } \\
\text { Size }\end{array}$ & $\begin{array}{l}\text { Follow-up } \\
\text { months(median/range) }\end{array}$ & $\begin{array}{l}\text { Clinical } \\
\text { stage }\end{array}$ & $\begin{array}{l}\text { Cut-off } \\
\text { value }\end{array}$ & Outcome & Cancer typi \\
\hline Sakin & 2019 & Turkey & Retrospective & $\begin{array}{l}61.3(22- \\
82)\end{array}$ & 115 & 16.2 & Advanced & 9 & OS & NSCLC \\
\hline Sakin & 2019 & Turkey & Retrospective & $59(42-83)$ & 90 & NA & Mixed & NA & OS & NSCLC \\
\hline Omar & 2018 & Turkey & Retrospective & NA & 496 & 33 & Advanced & 9.1 & OS, PFS & NSCLC \\
\hline Shi & 2018 & China & Retrospective & $\begin{array}{l}53.3(27- \\
73)\end{array}$ & 90 & NA & Advanced & 10.85 & OS & NSCLC \\
\hline Shi & 2018 & China & Retrospective & $57(44-72)$ & 79 & NA & Advanced & 9.3 & OS & NSCLC \\
\hline Gao & 2017 & China & Retrospective & $60(24-82)$ & 546 & 44.6 & Mixed & 11 & OS,DFS & NSCLC \\
\hline Cui & 2016 & China & Retrospective & $\begin{array}{l}57.3(32- \\
80)\end{array}$ & 270 & 60 & Mixed & NA & os & NSCLC \\
\hline Kumagai & 2014 & Japan & Retrospective & 69(19-87) & 308 & 36 & Mixed & 8.5 & OS,DFS & NSCLC \\
\hline Wang & 2019 & China & Retrospective & $60(27-80)$ & 101 & NA & Mixed & 10.282 & OS & NSCLC,SCL \\
\hline Hur & 2020 & Korea & Retrospective & NA & 116 & 60 & Mixed & 8.6 & OS,DFS & NSCLC \\
\hline Shen & 2019 & China & Retrospective & $60.96+-8.70$ & 138 & NA & Mixed & 10 & PFS & SCLC \\
\hline Watanabe & 2018 & Japan & Retrospective & $69(35-86)$ & 72 & 25.8 & Advanced & 10.3 & OS/PFS & NSCLC \\
\hline
\end{tabular}

Table 2

Newcastle Ottawa Scale for observational studies

\begin{tabular}{|llllll|}
\hline Author & Year & Selection (5) & Comparability (2) & Outcome (3) & Total(maximum = 10) \\
\hline Sakin et al.(a) & 2019 & 3 & 2 & 3 & 8 \\
\hline Sakin et al.(b) & 2019 & 3 & 2 & 3 & 8 \\
\hline Omar et al. & 2018 & 3 & 2 & 3 & 8 \\
\hline Shi et al. & 2018 & 3 & 2 & 3 & 8 \\
\hline Gao et al. & 2017 & 3 & 2 & 3 & 8 \\
\hline Cui et al. & 2016 & 2 & 2 & 3 & 7 \\
\hline Kumagai et al. & 2014 & 2 & 1 & 3 & 6 \\
\hline Wang et al. & 2019 & 3 & 2 & 3 & 8 \\
\hline Hur et al. & 2020 & 3 & 2 & 3 & 8 \\
\hline Shen et al. & 2019 & 3 & 2 & 3 & 8 \\
\hline Watanabe et al. & 2018 & 3 & 2 & 3 & 8 \\
\hline
\end{tabular}

\section{Meta-analysis (OS, DFS/PFS)}

Ten studies ${ }^{11,15,22-28,30}$ including 2283 patients were included in the analysis to show an association of MPV with OS. The pooled hazard ratio for OS was: 1.07 (95\% C.I.: 0.84-1.35), p value $=0.60$. As the analysis showed significant heterogeneity $\left(I^{2}=77.53 \%, p=<0.001\right)$, a random-effect model was used. This shows MPV values are not significantly associated with unfavorable OS among lung cancer patients. Furthermore, subgroup analysis was done as shown in Table 3 on different headings like analysis of variable, study region, sample size, clinical stage, cancer type, and cut-off also showed no significant association between MPV values and OS. 
Table 3

Subgroup Analysis for OS and DFS to show association with MPV.

\begin{tabular}{|c|c|c|c|c|c|c|c|}
\hline \multirow[t]{2}{*}{ Subgroup Analysis } & \multirow[b]{2}{*}{ Subgroups } & \multirow[b]{2}{*}{ No. of studies } & \multirow[b]{2}{*}{$\mathrm{HR}(95 \% \mathrm{Cl})$} & \multirow[b]{2}{*}{$\mathrm{p}$-value } & \multicolumn{2}{|c|}{ Heterogeneity } & \multirow[b]{2}{*}{ Model used } \\
\hline & & & & & 12 & $\mathrm{Ph}$ & \\
\hline \multirow[t]{19}{*}{ OS } & Analysis of variable & & & & & & \\
\hline & Multivariate & 6 & $1.07(0.69-1.68)$ & 0.76 & $84.74 \%$ & $<0.001$ & Random \\
\hline & Univariate & 5 & $1.12(1.00-1.27)$ & 0.06 & $0 \%$ & 0.68 & Fixed \\
\hline & Study Region & & & & & & \\
\hline & China & 5 & $0.93(0.59-1.48)$ & 0.77 & $84.86 \%$ & $<0.001$ & Random \\
\hline & Others & 6 & $1.20(0.88-1.62)$ & 0.24 & $71.95 \%$ & $<0.001$ & Random \\
\hline & Sample size & & & & & & \\
\hline & $<200$ & 7 & $0.98(0.87-1.11)$ & 0.79 & $36.38 \%$ & 0.15 & Fixed \\
\hline & $>200$ & 4 & $1.18(0.62-2.25)$ & 0.61 & $91.42 \%$ & $<0.001$ & Random \\
\hline & Clinical stage & & & & & & \\
\hline & Advanced & 5 & $1.26(0.81-1.97)$ & 0.31 & $68.39 \%$ & 0.01 & Random \\
\hline & Mixed & 6 & $0.98(0.71-1.36)$ & 0.91 & $84.20 \%$ & $<0.001$ & Random \\
\hline & Cancer Type & & & & & & \\
\hline & NSCLC & 10 & $1.09(0.84-1.41)$ & 0.53 & $79.75 \%$ & $<0.001$ & Random \\
\hline & NSCLC + SCLC & 1 & $0.95(0.64-1.41)$ & 0.79 & NA & NA & Random \\
\hline & SCLC & - & & & & & \\
\hline & Cut-off value & & & & & & \\
\hline & $<10$ & 5 & $1.18(0.82-1.69)$ & 0.37 & $68.38 \%$ & 0.01 & Random \\
\hline & $>=10$ & 4 & $0.82(0.45-1.48)$ & 0.51 & $74.55 \%$ & 0.01 & Random \\
\hline \multirow[t]{19}{*}{ DFS/PFS } & Analysis of variable & & & & & & \\
\hline & Multivariate & 4 & $1.25(0.55-2.80)$ & 0.59 & $90.91 \%$ & $<0.001$ & Random \\
\hline & Univariate & 2 & $0.81(0.71-0.93)$ & $<0.001$ & $0 \%$ & 0.84 & Fixed \\
\hline & Study Region & & & & & & \\
\hline & China & 2 & $0.63(0.36-1.09)$ & 0.1 & $89.52 \%$ & $<0.001$ & Random \\
\hline & Others & 4 & $1.53(1.14-2.03)$ & $<0.001$ & $42.11 \%$ & 0.16 & Fixed \\
\hline & Sample size & & & & & & \\
\hline & $<200$ & 3 & $1.04(0.60-1.82)$ & 0.88 & $76.40 \%$ & 0.01 & Random \\
\hline & $>200$ & 3 & $1.07(0.41-2.81)$ & 0.89 & $92.21 \%$ & $<0.001$ & Random \\
\hline & Clinical stage & & & & & & \\
\hline & Advanced & 2 & $1.84(1.19-2.82)$ & 0.01 & $0 \%$ & 0.68 & Fixed \\
\hline & Mixed & 4 & $0.82(0.52-1.30)$ & 0.4 & $85.38 \%$ & $<0.001$ & Random \\
\hline & Cancer Type & & & & & & \\
\hline & NSCLC & 5 & $1.13(0.58-2.20)$ & 0.71 & $88.01 \%$ & $<0.001$ & Random \\
\hline & SCLC & 1 & $0.81(0.71-0.93)$ & $<0.001$ & NA & NA & Random \\
\hline & NSCLC + SCLC & - & & & & & \\
\hline & Cut-off value & & & & & & \\
\hline & $<10$ & 3 & $1.35(0.83-2.19)$ & 0.23 & $51.62 \%$ & 0.13 & Random \\
\hline & $>=10$ & 3 & $0.86(0.48-1.54)$ & 0.61 & $89.76 \%$ & $<0.001$ & Random \\
\hline
\end{tabular}


Similarly, six studies ${ }^{11,23,25,28-30}$ including 1676 patients were included in an analysis to show an association of MPV values with DFS/PFS of lung cancer patients. The pooled HRs for MPV levels were 1.04 (95\% C.I.:0.68-1.60), p-value: 0.84 indicating no significant association between MPV level and DFS/PFS in lung cancer patients. The analysis showed significant heterogeneity $\left(I^{2}=85.38 \%\right.$, $\mathrm{P}$ value $\left.=<0.001\right)$, so a random effect model was used. Subgroup analysis was done as shown in Table 3 and stratified in different subgroups like analysis of variable, study region, sample size, clinical stage, cancer type, and cut-off values. Under the analysis of variables, studies with univariate analysis showed a significant association between low MPV levels and worse DFS/PFS. (HR = $0.81,95 \% \mathrm{Cl}$. $=0.71-0.93$, p-value $<0.001)$ with no heterogeneity. Similarly, studies conducted in countries other than China (HR:1.53, 95\% C.I. $=1.14-2.03, \mathrm{p}-$ value $<0.001, I 2=42.11 \%$ ) and studies with advanced-stage lung cancer patients (HR: $1.84,95 \%$ C.I. $=1.19-2.82, p$-value $=0.01, I 2=0 \%$ ) showed significant association between MPV levels and worse DFS/PFS. While other subgroups showed no significant association.

\section{Publication bias and Sensitivity analysis}

Figure 4 shows a symmetrical funnel plot and egger's regression test with $p=0.1184$ suggesting no significant publication among studies included for the analysis of OS. For a similar reason,

There is no evidence of publication bias among studies included in the analysis of DFS/PFS [egger's regression test $(p=0.2995)$ ]. (Fig. 5)

Sensitivity analysis done for studies included in OS and DFS/PFS showed the reliability and robustness of our analysis. (Figs. 6 and 7)

\section{Discussion}

The investigations and research on prognostic markers are very crucial particularly among cancer patients as they aid in the improvement of existing and development of newer treatment therapies and patient care. Previous meta-analyses have only analyzed survival outcomes with different types of cancers including lung cancer. To our knowledge, this is the first meta-analysis to assess the prognostic significance of MPV with survival outcome among lung cancer patients only.

A meta-analysis by Pyo et al. in 2016 showed that MPV levels in patients with malignant tumors were significantly higher than in healthy subjects. However, in lung cancer, the MPV of pre-treatment patients was lower than that of healthy subjects but not statistically significant (mean difference - $0.352,95 \% \mathrm{Cl}-$ 0.763 to 0.060$).{ }^{13} \mathrm{~A}$ recent meta-analysis found no significant association between MPV levels and survival outcomes among cancer patients. While doing subgroup analysis including 7 studies, higher MPV levels were not associated with worse OS (NSCLC: HR $0.85,95 \%$ Cl 0.64 to 1.15 )..$^{14}$ Our study included 11 studies with NSCLC, SCLC, and NSCLC + SCLC, and similar to the above studies showed no statistically significant association.

Subgroup analysis was conducted by country of origin, cut-off value, tumor stage and tumor type, type of analysis. All the subgroups showed insignificant association with MPV values and OS. While in DFS, a significant association was shown between low MPV and worse DFS in a univariate analysis. But we believe this result requires verifying the prognostic significance of a univariate in the validation cohort since the univariate analysis actually has a high risk of bias leading to overestimation of sensitivity and specificity in predicting cancer prognosis. Interestingly, worse DFS was shown to be significantly associated with MPV values among advanced cancer patients. These findings are similar to a study showing MPV levels in patients in an early-stage lung cancer similar to those found in healthy subjects and increased with the cancer progression. ${ }^{31}$ However, the result cannot be generalized considering few studies and sample size.

Platelet volume is determined during both megakaryopoiesis and thrombopoiesis. Various stages of platelet production and maturation are influenced by cytokines like interleukin-6 (IL-6), granulocyte colony-stimulating factor (G-CSF), and macrophage colony-stimulating factor (M-CSF). ${ }^{32}$ Moreover, platelets can get activated on encountering circulating tumor cells which result in the formation of microparticles that can potentially promote the invasiveness of tumor cells. ${ }^{33}$ Therefore, this close interplay between high MPV and poor prognosis of cancers can be a reasonable hypothesis. In contrast, our findings do not support the hypothesis that an MPV level is a prognostic factor for poor outcomes in lung cancer patients.

The strength of our study lies in the fact that this is the first meta-analysis to show the association between MPV levels and survival outcomes in lung cancer patients. In contrast to previously published meta-analyses, our study results enable a deeper comprehensive understanding of the predictive role of MPV in lung cancer. However, there are still several limitations in our work. The inclusion of retrospective studies and inclusion of studies published in the English language only might have added biases of any form in our study and excluded probable other studies. Second, studies provided HRs and $95 \%$ Cls from univariable analyses, which could lead to bias towards the overestimation of the prognostic role of the MPV, as the HR in multivariable analyses may not be statistically significant after the consideration of other elements. In addition, as with all meta-analyses, heterogeneity resulting from various factors as depicted in the subgroup analysis may have the potential to affect the interpretation of the results. Lastly, the clinical application of MPV in predicting DFS mandates further verification due to the lack of a standardized cut-off value.

\section{Conclusion}

Our meta-analysis showed pretreatment MPV levels don't have any prognostic significance except in advanced lung cancer cases. Further high-quality, welldesigned, large-scale studies with uniform cut-off value are necessary for establishing the role of MPV as a prognostic tool for screening and/or monitoring of lung cancer in clinical practice.

\section{Abbreviations}

MPV $=$ Mean Platelet Volume 
OS= Overall Survival

DFS/PFS= Disease Free Survival/ Progression Free Survival

NSCLC $=$ Non-small Cell Lung Cancer

SCLC $=$ Small Cell Lung Cancer

NOS $=$ Newcastle Ottawa Scale

$\mathrm{HR}=$ Hazards Ratio

G-CSF= Granulocyte Colony-Stimulating Factor

M-CSF= Macrophage Colony-Stimulating Factor

\section{Declarations}

Acknowledgment: None

Competing Interest: None to declare.

Availability of data and Materials: All the necessary data and information are within the article. Supplementary file with the search strategy has been provided.

Funding: No funding was required for the work.

Ethical approval and Consent to participate: Not applicable.

Consent for publication: Not applicable

Authors Contributions: SK and SS were involved in conceptualization of the study along with designing the study search strategy, reviewed study abstracts, extracted data from full text articles, and drafted the initial manuscript. PS, RR and RS were involved in editing and revising the manuscript. All the authors read and approved the final version of the manuscript.

\section{Corresponding Author}

Correspondence to Sanjeev Kharel

Author Affiliation

Maharajgunj Medical Campus, Tribhuvan University Institute of Medicine, Kathmandu, Nepal

Sanjeev Kharel, Suraj Shrestha

\section{National Cancer Hospital and Research Center, Harisiddhi, Lalitpur, Nepal}

Prafulla Shakya

Nepalese Army Institute of Health Science, College of Medicine, Kathmandu, Nepal

Rohit Rawat

Department of Clinical Oncology, National Academy of Medical Science, Kathmandu, Nepal

Ramila Shilpakar

\section{References}

1. Siegel RL, Miller KD, Jemal A. Cancer statistics. 2018. CA Cancer J Clin. 2018 Jan;68(1):7-30.

2. Vijayvergia N, Shah PC, Denlinger CS. Survivorship in Non-Small Cell Lung Cancer: Challenges Faced and Steps Forward. J Natl Compr Canc Netw. 2015 Sep;13(9):1151-61.

3. Greenberg AK, Lee MS. Biomarkers for lung cancer: clinical uses. Curr Opin Pulm Med. 2007 Jul;13(4):249-55.

4. Gasparyan AY, Ayvazyan L, Mikhailidis DP, Kitas GD. Mean platelet volume: a link between thrombosis and inflammation? Curr Pharm Des. 2011;17(1):47-58.

5. Riedl J, Kaider A, Marosi C, Prager G, Eichelberger B, Koder S, et al. PO-63 - Exhausted platelets in cancer patients with high risk of venous thromboembolism and poor prognosis. Thromb Res. 2016 Apr;140Suppl 1:S199-200.

6. Boneu B, Bugat R, Boneu A, Eche N, Sie P, Combes PF. Exhausted platelets in patients with malignant solid tumors without evidence of active consumption coagulopathy. Eur J Cancer Clin Oncol. 1984 Jul;20(7):899-903. 
7. Shen X-M, Xia Y-Y, Lian L, Zhou C, Li X-L, Han S-G, et al. Mean platelet volume provides beneficial diagnostic and prognostic information for patients with resectable gastric cancer. Oncol Lett. 2016 Oct;12(4):2501-6.

8. Wang X, Cui M-M, Xu Y, Liu L, Niu Y, Liu T, et al. Decreased mean platelet volume predicts poor prognosis in invasive bladder cancer. Oncotarget. 2017 Sep 15;8(40):68115-22.

9. Oge T, Yalcin OT, Ozalp SS, Isikci T. Platelet volume as a parameter for platelet activation in patients with endometrial cancer. J Obstet Gynaecol. 2013 Apr;33(3):301-4.

10. Yun Z-Y, Zhang X, Liu Z-P, Liu T, Wang R-T, Chen H. Association of decreased mean platelet volume with renal cell carcinoma. Int J Clin Oncol. 2017 Dec;22(6):1076-80.

11. Kumagai S, Tokuno J, Ueda Y, Marumo S, Shoji T, Nishimura T, et al. Prognostic significance of preoperative mean platelet volume in resected non-smallcell lung cancer. Mol Clin Oncol. 2015 Jan;3(1):197-201.

12. Scheiner B, Kirstein M, Popp S, Hucke F, Bota S, Rohr-Udilova N, et al. Association of Platelet Count and Mean Platelet Volume with Overall Survival in Patients with Cirrhosis and Unresectable Hepatocellular Carcinoma. Liver Cancer. 2019 May;8(3):203-17.

13. Pyo J-S, Sohn JH, Kang G. Diagnostic and prognostic roles of the mean platelet volume in malignant tumors: a systematic review and meta-analysis. Platelets. 2016 Dec;27(8):722-8.

14. Chen X, Li J, Zhang X, Liu Y, Wu J, Li Y, et al. Prognostic and clinicopathological significance of pretreatment mean platelet volume in cancer: a metaanalysis. BMJ Open. 2020 Oct 27;10(10):e037614.

15. Sakin A, Secmeler S, Arici S, Geredeli C, Yasar N, Demir C, et al. Prognostic Significance of Mean Platelet Volume on Local Advanced Non-Small Cell Lung Cancer Managed with Chemoradiotherapy. Sci Rep. 2019 Mar 8;9(1):3959.

16. Perry R, Taylor M, Lewis L, Yellowlees A, Fleetwood K, Barata T. Estimating Survival Data from Published Kaplan-Meier Curves: a Comparison of Methods. Value Health. 2014 Nov;17(7):A326.

17. Newcastle-Ottawa scale for. assessment of quality of included studies-Cohort studies. Available from: http://dx.doi.org/10.7717/peerj.8815/supp-4.

18. Higgins JPT, Thompson SG, Deeks JJ, Altman DG. Measuring inconsistency in meta-analyses. BMJ. 2003 Sep 6;327(7414):557-60.

19. Kontopantelis E, Reeves D. Performance of statistical methods for meta-analysis when true study effects are non-normally distributed: A simulation study. Stat Methods Med Res. 2012 Aug;21(4):409-26.

20. Begg CB, Mazumdar M. Operating Characteristics of a Rank Correlation Test for Publication Bias. Vol. 50, Biometrics. 1994. p. 1088. Available from: http://dx.doi.org/10.2307/2533446.

21. Egger M, Smith GD, Schneider M, Minder C. Bias in meta-analysis detected by a simple, graphical test. Vol. 315, BMJ. 1997. p. 629-34. Available from: http://dx.doi.org/10.1136/bmj.315.7109.629.

22. Sakin A, Yasar N, Arici S, Demir C, Geredeli C, Aksaray F, et al. Effect of Pretreatment Platelet Parameters on Survival in Limited Disease Small Cell Lung Cancer. Asian Pac J Cancer Prev. 2019 Jun;20(6)(1):1879-85.

23. Omar M, Tanriverdi O, Cokmert S, Oktay E, Yersal O, Pilancı KN, et al. Role of increased mean platelet volume (MPV) and decreased MPV/platelet count ratio as poor prognostic factors in lung cancer. Clin Respir J. 2018 Mar;12(3):922-9.

24. Shi L, Li Y, Yu T, Wang Z, Zhou C, Xing W, et al. Predictable Resistance and Overall Survival of Gemcitabine/Cisplatin by Platelet Activation Index in NonSmall Cell Lung Cancer. Med Sci Monit. 2018 Nov;30:24:8655-68.

25. Gao L, Zhang H, Zhang B, Zhang L, Wang C. Prognostic value of combination of preoperative platelet count and mean platelet volume in patients with resectable non-small cell lung cancer. Oncotarget. 2017 Feb 28;8(9):15632-41.

26. Cui M-M, Li N, Liu X, Yun Z-Y, Niu Y, Zhang Y, et al. Platelet distribution width correlates with prognosis of non-small cell lung cancer. Sci Rep. 2017 Jun;14(1):3456. 7(.

27. Wang J-J, Wang Y-L, Ge X-X, Xu M-D, Chen K, Wu M-Y, et al. Prognostic Values of Platelet-Associated Indicators in Resectable Lung Cancers. Technol Cancer Res Treat. 2019 Jan;1:18:1533033819837261.

28. Hur JY, Lee HY, Chang HJ, Choi CW, Kim DH, Eo WK. Preoperative plateletcrit is a Prognostic Biomarker for Survival in Patients with Non-Small Cell Lung Cancer. J Cancer. 2020 Feb;25(10):2800-7. 11(.

29. Shen X-B, Wang Y, Shan B-J, Lin L, Hao L, Liu Y, et al. Prognostic Significance Of Platelet-To-Lymphocyte Ratio (PLR) And Mean Platelet Volume (MPV) During Etoposide-Based First-Line Treatment In Small Cell Lung Cancer Patients. Cancer Manag Res. 2019 Oct 17;11:8965-75.

30. Watanabe K, Yasumoto A, Amano Y, Kage H, Goto Y, Yatomi Y, et al. Mean platelet volume and lymphocyte-to-monocyte ratio are associated with shorter progression-free survival in EGFR-mutant lung adenocarcinoma treated by EGFR tyrosine kinase inhibitor. PLoS One. 2018 Sep;7(9):e0203625. 13(.

31. Korniluk A, Koper-Lenkiewicz OM, Kamińska J, Kemona H, Dymicka-Piekarska V. Mean Platelet Volume (MPV): New Perspectives for an Old Marker in the Course and Prognosis of Inflammatory Conditions. Mediators Inflamm. 2019 Apr 17;2019:9213074.

32. Kaushansky K. Growth factors and hematopoietic cell fate. A new feature: controversies in hematology. Blood. 1998 Jul 15;92(2):345-344.

33. Delago D, Knittelfelder O, Jakse G, Lukasiak K, Reinisch S, Renner W, et al. The decreased mean platelet volume is associated with poor prognosis in patients with oropharyngeal cancer treated with radiotherapy. Radiat Oncol. 2020 Nov;7(1):259. 15.

\section{Figures}




\section{PRISMA Flow Diagram}

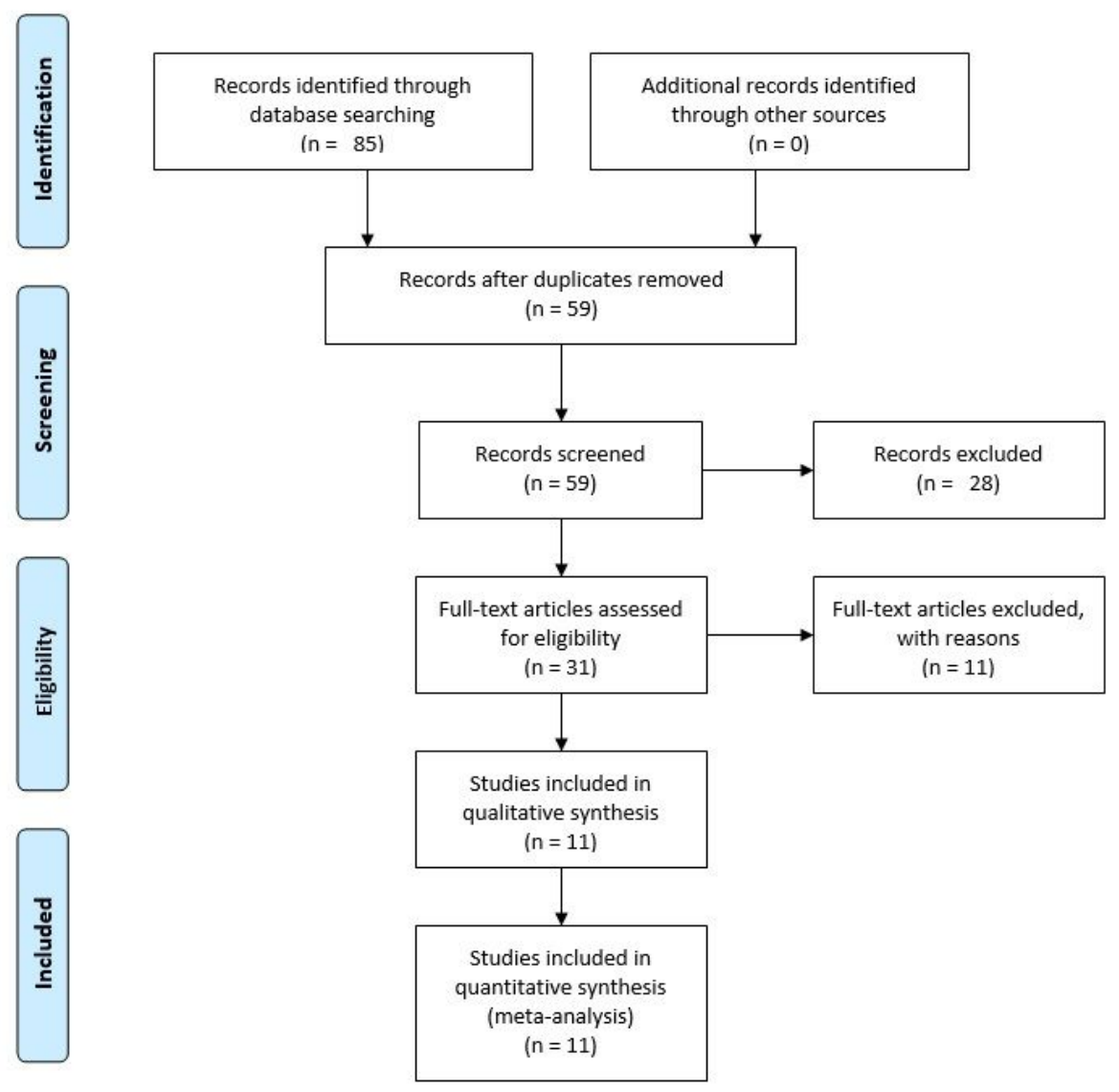

Figure 1

A flowchart demonstrating the details of the study selection according to the PRISMA guidelines

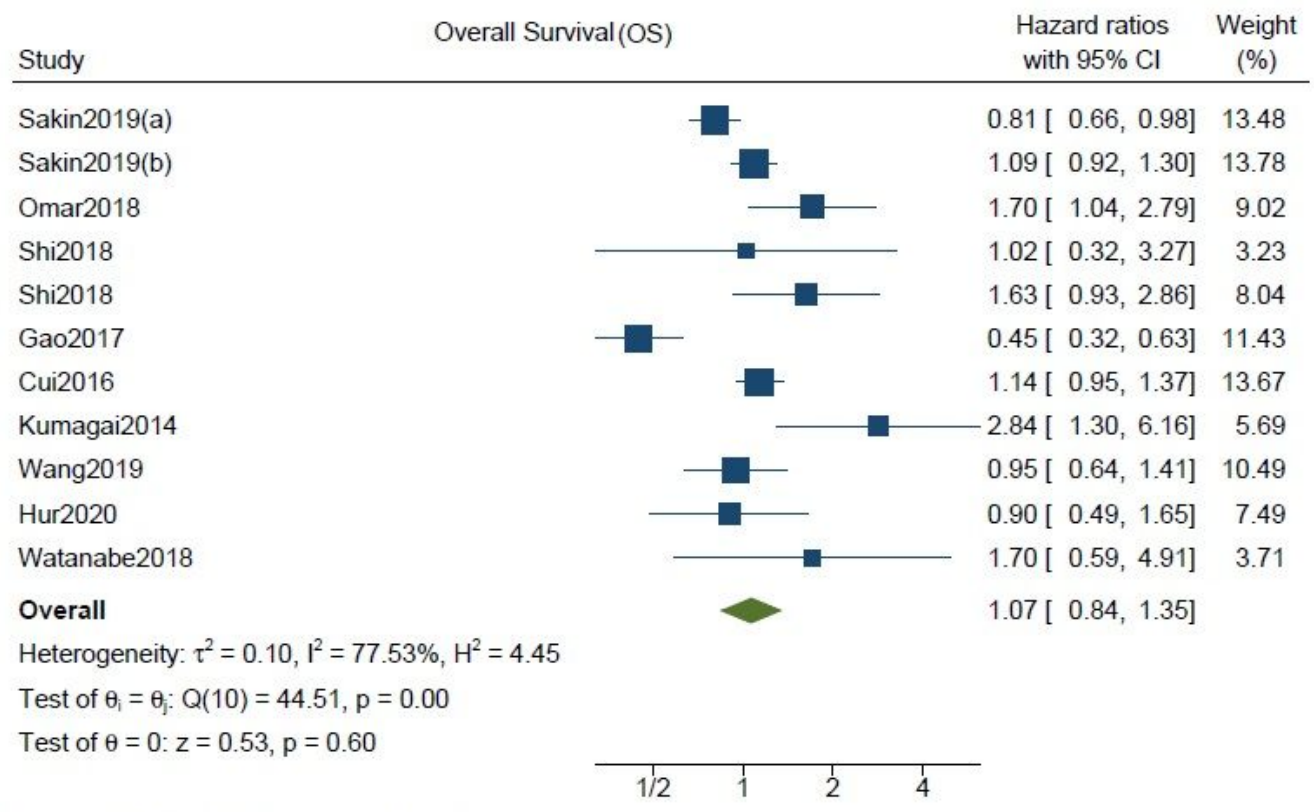

Random-effects DerSimonian-Laird model

Figure 2

Forest plot with $95 \% \mathrm{Cl}$ for meta-analysis to show association between OS and MPV. 


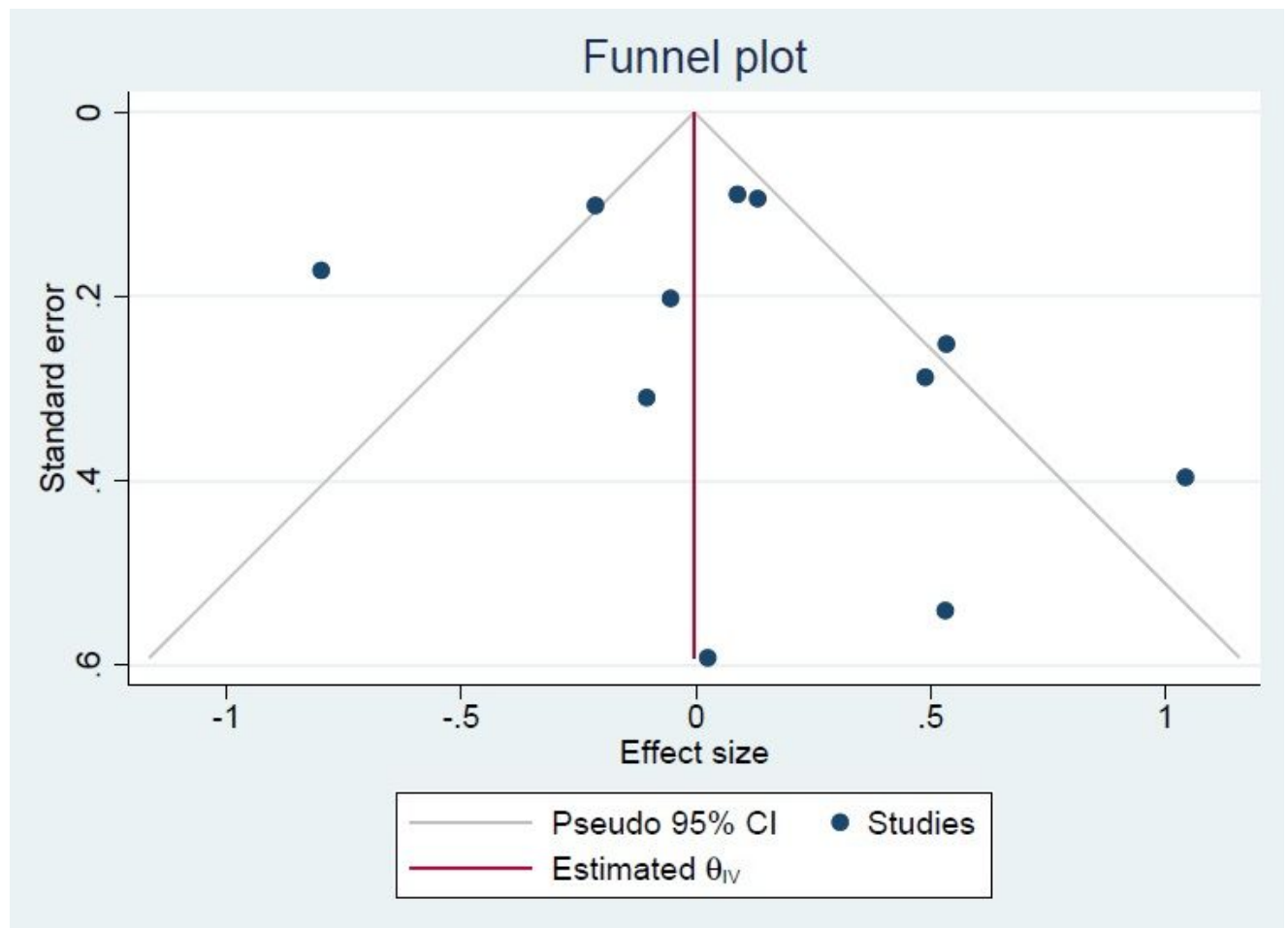

Figure 3

Funnel plot for OS of four studies.

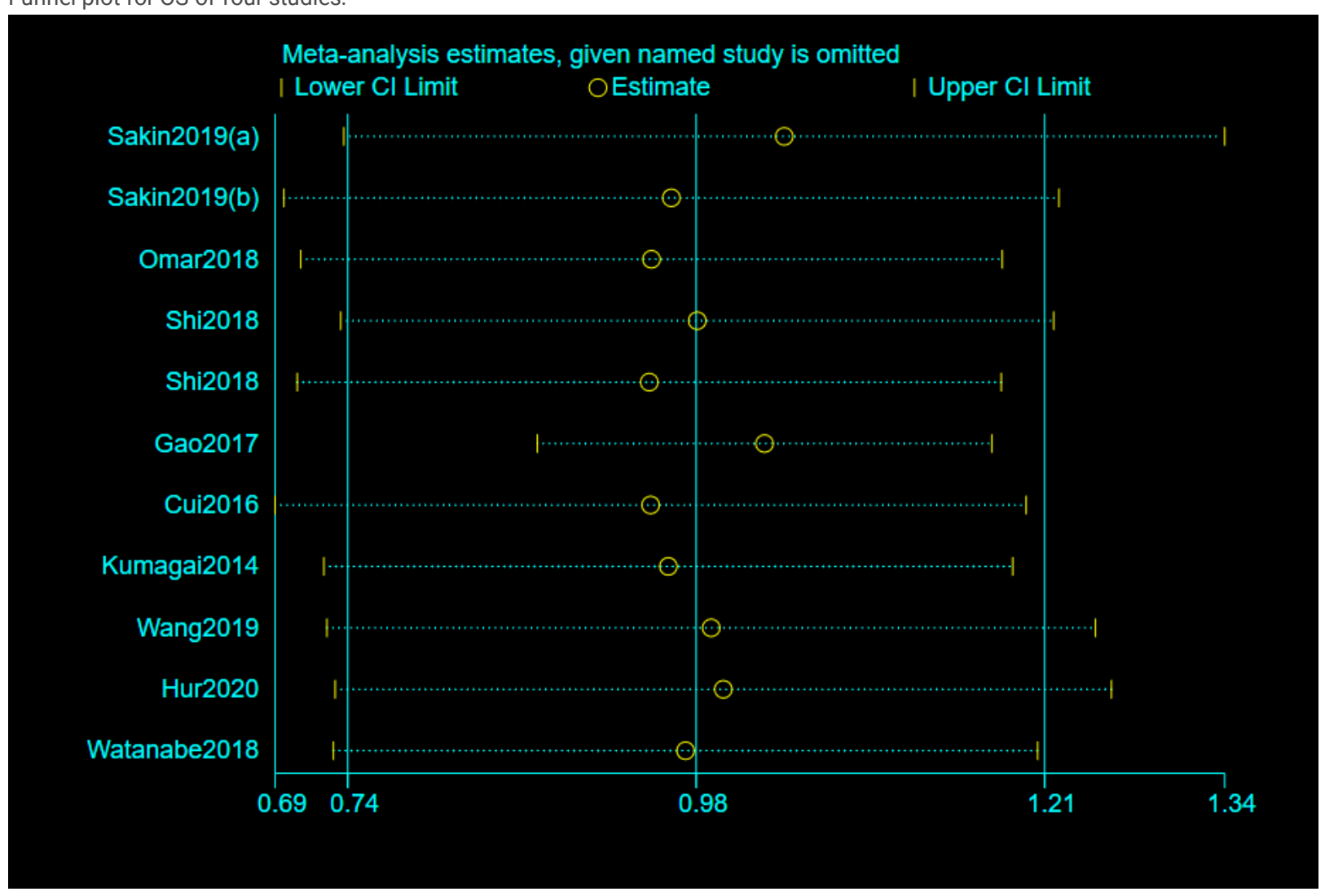

Figure 4

Sensitivity analysis for studies for OS. 


\begin{tabular}{|c|c|c|c|}
\hline Study & $\begin{array}{l}\text { Disease Free Survival/Progression Free Survival } \\
\text { (DFS/PFS) }\end{array}$ & $\begin{array}{l}\text { Hazard ratios } \\
\text { with } 95 \% \mathrm{Cl}\end{array}$ & $\begin{array}{l}\text { Weight } \\
(\%)\end{array}$ \\
\hline Omar2018 & & $1.67[0.89,3.12]$ & 14.56 \\
\hline Gao2017 & -2 & $0.46[0.33,0.64]$ & 18.80 \\
\hline Kumagai2014 & & $1.71[1.07,2.74]$ & 16.89 \\
\hline Hur2020 & a & $0.76[0.39,1.50]$ & 13.83 \\
\hline Shen2019 & & $0.81[0.71,0.93]$ & 20.85 \\
\hline Watanabe2018 & & $-2.00[1.11,3.62]$ & 15.06 \\
\hline Overall & & $1.04[0.68,1.60]$ & \\
\hline \multicolumn{4}{|c|}{ Heterogeneity: $\tau^{2}=0.22, \mathrm{I}^{2}=85.38 \%, \mathrm{H}^{2}=6.84$} \\
\hline \multicolumn{4}{|c|}{ Test of $\theta_{\mathrm{i}}=\theta_{\mathrm{j}}: \mathrm{Q}(5)=34.20, \mathrm{p}=0.00$} \\
\hline \multicolumn{4}{|c|}{ Test of $\theta=0: z=0.20, p=0.84$} \\
\hline & $1 / 2$ & & \\
\hline
\end{tabular}

Figure 5

Forest plot with $95 \% \mathrm{Cl}$ for meta-analysis to show association between DFS/PFS and MPV.

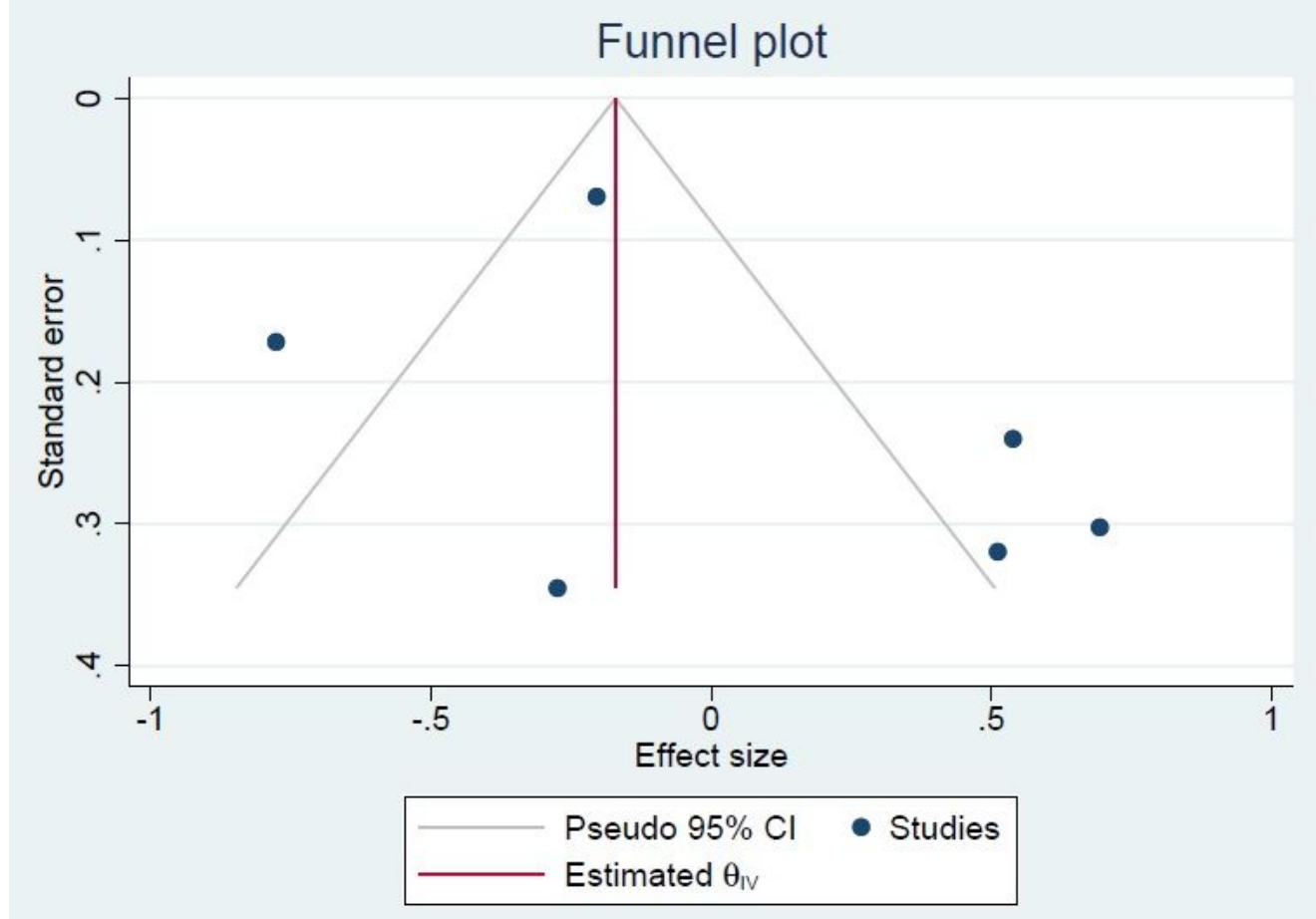

Figure 6

Funnel plot for DFS/PFS of four studies. 


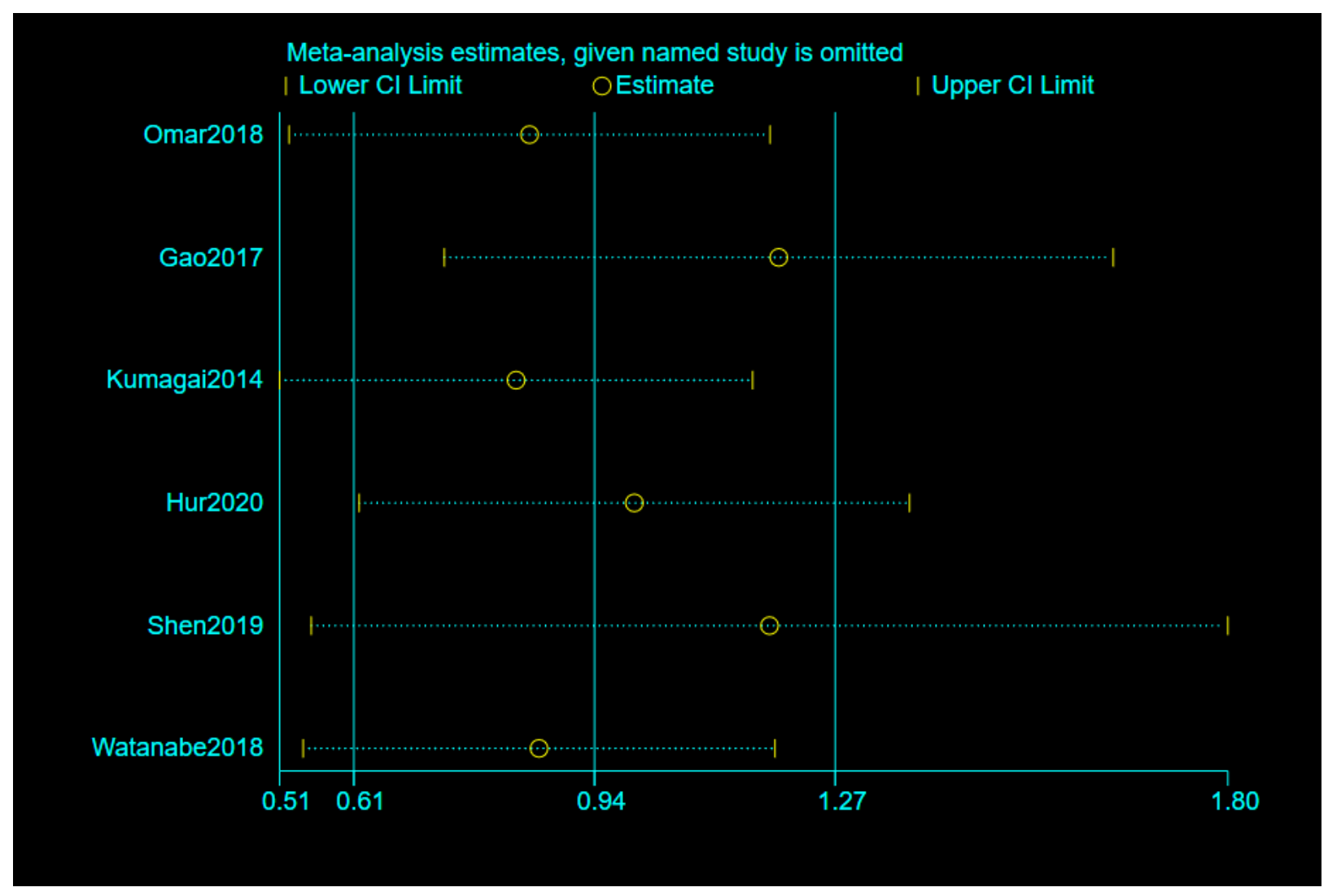

Figure 7

Sensitivity analysis for studies of DFS/PFS

\section{Supplementary Files}

This is a list of supplementary files associated with this preprint. Click to download.

- Additionallnformation.docx 